\title{
Simulation of neutron production in hadron-nucleus and nucleus-nucleus interactions in Geant4
}

\author{
Aida Galoyan ${ }^{1, *}$, Alberto Ribon ${ }^{2}$, and Vladimir Uzhinsky ${ }^{1,2}$ \\ ${ }^{1}$ JINR, Dubna, Russia \\ ${ }^{2}$ CERN, Geneva, Switzerland
}

\begin{abstract}
Studying experimental data obtained at ITEP [1] on neutron production in interactions of protons with various nuclei in the energy range from 747 $\mathrm{MeV}$ up to $8.1 \mathrm{GeV}$, we have found that slow neutron spectra have scaling and asymptotic properties [2]. The spectra weakly depend on the collision energy at momenta of projectile protons larger than $5-6 \mathrm{GeV} / \mathrm{c}$. These properties are taken into account in the Geant4 Fritiof (FTF) model. The improved FTF model describes as well as the Geant4 Bertini model the experimental data on neutron production by $1.2 \mathrm{GeV}$ and $1.6 \mathrm{GeV}$ protons on targets $(\mathrm{Fe}, \mathrm{Pb})[3]$ and by $3.0 \mathrm{GeV}$ protons on various targets $(\mathrm{Al}, \mathrm{Fe}, \mathrm{Pb})[4]$. For neutron production in antiproton-nucleus interactions, it is demonstrated that the FTF results are in a satisfactory agreement with experimental data of the LEAR collaboration [5]. The FTF model gives promising results for neutron production in nucleus - nucleus interactions at projectile energy $1-2 \mathrm{GeV}$ per nucleon [6]. The observed properties allow one to predict neutron yields in the nucleus-nucleus interactions at high and very high energies. Predictions for the NICA/MPD experiment at JINR are presented.
\end{abstract}

\section{Introduction}

For Beam-Energy Scan program running now at RHIC (BES), and new experiments at future accelerator complexes - such as FAIR at GSI and NICA at JINR - it is very important to know the yield of neutrons for detector design, estimations of radiation doses, creation of Zero Degree and hadron calorimeters. For these reasons, it is needed to verify and develop Monte Carlo generators for neutron production in hadron-nucleus and nucleus-nucleus interactions. There are some generators - UrQMD [7, 8], LAQGSM [9], Fluka[10] and Geant4 [11] models, which are able to simulate nucleus-nucleus interactions.

The standard UrQMD model does not treat the nuclear remnant. Since the source codes of LAQGSM and Fluka are not publicly available, we use and develop Geant4 FTF generator, whose source code is open. We also apply Geant 4 Bertini and Binary Cascade models for calculations and comparisons with the FTF results and experimental data. The Geant4 FTF model is based on the Fritiof model $[12,13]$ of the LUND university. Description of the Geant4 FTF model is given in the paper [11].

We have performed an analysis of a large set of ITEP experimental data [1] on neutron production with energies from 7.5 up to $190 \mathrm{MeV}$ in proton-nucleus interactions. Asymptotical and scaling properties of produced neutrons have been found. We used these properties

\footnotetext{
*e-mail: galoyan@1xpub01.jinr.ru
} 
of neutron spectra to develop the Geant4 FTF model. Then, the experimental data on neutron production for nuclear reactions induced by protons at 1.2, $1.6 \mathrm{GeV}$ [3], and 3.0 GeV [4] for various targets have been considered. We have tried to describe these experimental data using the improved FTF, Bertini and Binary cascade models. Experimental data on neutron production in anti-proton - nucleus interactions of the LEAR collaboration [5] have been also studied. We applied calculations with FTF+Preco (FTFP) and FTF+Binary cascade (FTFB) models for comparison with the LEAR experimental data. For the FTF model verification we used also experimental data on neutron production in collisions of light nuclei with nuclei at 1 and $2 \mathrm{GeV}$ per nucleon. The obtained results allow us to give FTF model predictions for the future experiment MPD at the new accelerator NICA at JINR.

\section{Main assumptions and development of the FTF model}

The main assumptions of the Fritiof model are quite simple. It is assumed that hadrons turn into excited states in hadron-hadron collisions. If only one hadron is excited, the process is called diffraction dissociation. Excited states of hadrons are characterized by their respective masses. Minimal masses of excited states are different in various models. In the original Fritiof, the minimal mass is $1.2 \mathrm{GeV}$, in UrQMD is $1.46 \mathrm{GeV}$, and in Hijing is $2 \mathrm{GeV}$. In the Geant4 FTF model, we decreased the minimal mass of excited states to $1.16 \mathrm{GeV}$. This reflects on simulation of events with small multiplicity, in particular, in description of the diffraction dissociation. The excited states of hadrons are considered as quark-gluon strings. The Lund fragmentation model is used for quark-gluon string fragmentations. Neutrons are produced at the fragmentation of the quark-gluon strings, in the diffraction dissociation and in proton charge-exchange processes.

For simulation of hadron-nucleus and nucleus-nucleus interactions, the Glauber approach is applied in the FTF model to determine the number of nucleons participating in inelastic interactions. After that, string creation and fragmentation are simulated. The Glauber approach implemented in FTF is not sufficient for description of the observed destruction of nuclei. Thus, a reggeon cascading model [14] of nuclear destruction is applied in the FTF model, which plays a very important role in the production of nucleons. The model, inspired by Reggeon theory, assumes that spectator nucleons can be involved by the participating nucleons at the first stage of the interaction. A probability to involve a spectator nucleon having coordinates in the impact parameter plane $\overrightarrow{b_{i}}$ by a participating nucleon with coordinates $\overrightarrow{b_{j}}$ is written as:

$$
P_{i j}=C_{n d} e^{-\left(\overrightarrow{b_{i}}-\overrightarrow{b_{j}}\right)^{2} / R^{2}}, R \simeq 1.2(\mathrm{fm}),
$$

$C_{n d}$ is the parameter of nuclear destruction, given by the formula:

$$
C_{n d}=0.0048 \cdot A \cdot e^{4\left(y_{p}-2.1\right)} /\left(1+e^{4\left(y_{p}-2.1\right)}\right),
$$

where $\mathrm{A}$ is the mass number of the target nucleus and $y_{p}$ is the rapidity of the projectile in the target nucleus rest frame.

In the FTF model, momenta of participating and involved nucleons are sampled according to the expression:

$$
\begin{gathered}
P\left(p_{z}, \overrightarrow{p_{T}}\right) \propto e^{-\left|\overrightarrow{p_{T}}\right|^{2} /\left\langle{\overrightarrow{p_{T}}}^{2}\right\rangle} \cdot e^{-\left(x^{-}-1 / N\right)^{2} /(0.3 / N)^{2}}, \\
x^{-}=\left(E-p_{z}\right) /\left(E_{N}-P_{N}\right), \\
\left\langle\mid{\overrightarrow{p_{T}}}^{2}\right\rangle=0.035+0.04 \cdot e^{4\left(y_{p}-2.5\right)} /\left(1+e^{4\left(y_{p}-2.5\right)}\right),
\end{gathered}
$$


where $N$ is multiplicity of the involved and participating nucleons, $x^{-}$is the light-cone momentum fraction, $E_{N}$ and $P_{N}$ are the total energy and the momentum of all interacting nucleons of the target nucleus. Numbers in Eqs. (1)-(4) were determined [2] in order to reproduce the experimental data [1].

\section{Analysis of experimental data on neutron production}

Studying the experimental data on neutron production in the interactions of protons at energies from $747 \mathrm{MeV}$ up to $8.1 \mathrm{GeV}$ with various targets [1], we noted [2] that neutron spectra have interesting regularities. We found that slow and fast neutron production cross section are weakly energy-dependent at $P_{l a b}>5-6 \mathrm{GeV} / \mathrm{c}$ at neutron emission angle $119^{0}$. In other words, neutron spectra approach the asymptotic regime at $P_{l a b}>5 \mathrm{GeV} / \mathrm{c}$.
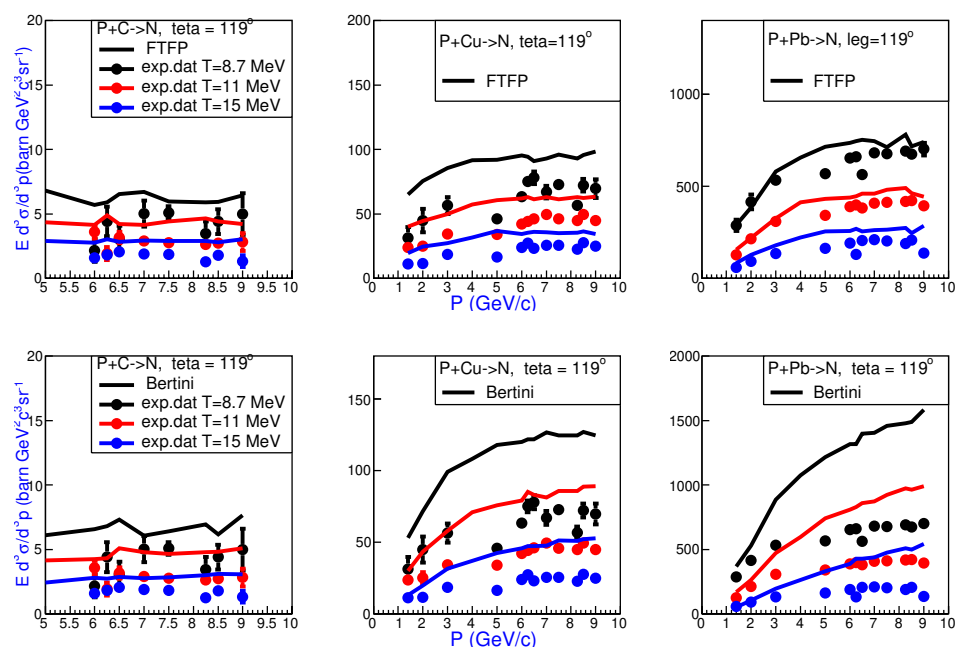

Figure 1. Inclusive cross sections of neutrons produced in $\mathrm{pC}, \mathrm{pCu}, \mathrm{pPb}$ interactions at angle 119 degrees as functions of projectile momenta. Points are experimental data [1]. Lines are calculations by the FTFP model in upper figures, and the Bertini model in bottom figures. Black points and lines correspond to neutrons with energy $8.7 \mathrm{MeV}$, red points and lines correspond to neutrons with energy $11 \mathrm{MeV}$, blue points and lines correspond to neutrons with energy $15 \mathrm{MeV}$

Let us consider the energy and target mass dependencies of the slow neutron cross sections at neutron kinetic energies $\mathrm{T}=8.5,11$, and $15 \mathrm{MeV}$. We believe that at these energies the data represent the main properties of the slow neutron spectra. The corresponding experimental data are presented in Fig. 1 for proton interactions with $\mathrm{C}, \mathrm{Cu}$, and $\mathrm{Pb}$ targets. Calculations by the FTF and Bertini models of the Geant4 are also shown in Fig. 1. As seen, for the lead and copper nuclei, the asymptotic behaviour takes place at $P_{\text {lab }} \sim 5 \mathrm{GeV} / \mathrm{c}$. Since the experimental data for the carbon nuclei start at projectile momenta $6 \mathrm{GeV} / \mathrm{c}$, only asymptotic regime for the carbon nuclei is seen at $P_{l a b} \geq 6 \mathrm{GeV} / \mathrm{c}$. The FTF results for $\mathrm{p}+\mathrm{C}$ and $\mathrm{p}+\mathrm{Cu}$ interactions are closer to the experimental data than the Bertini results. The Bertini model produces too many slow neutrons in the backward hemisphere at 119 degrees. For the lead target, the FTF model describes the experimental data better than the Bertini model does. According to the nuclear scaling hypothesis, the inclusive cross sections of nucleons scale as $A^{n}$, where $A$ is the mass number of target. The analysis of the experimental data [1] 
demonstrates [2] that the scaling coefficient of the evaporated neutron inclusive cross sections depends on the kinetic energy and can be presented as $A^{n} f(E)$.

Let us consider the experimental data on neutron production in proton interactions with $\mathrm{Al}, \mathrm{Fe}, \mathrm{Pb}$ at energy of projectile protons of $3 \mathrm{GeV}$, presented in paper [4]. In Fig. 2, inclusive cross sections of neutrons produced in $\mathrm{p}+\mathrm{Fe}$ interactions are shown at various angles of emitted neutrons: 15, 30, 60, 90, 120 and 150 degrees.
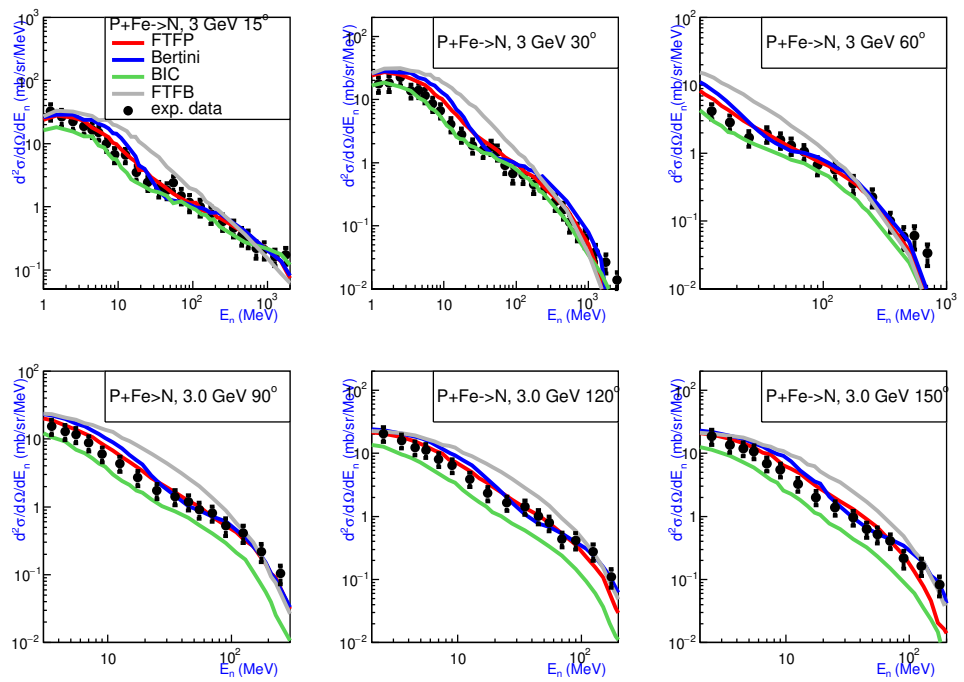

Figure 2. Inclusive cross sections of neutrons produced in $\mathrm{p}+\mathrm{Fe}$ interactions at projectile momentum 3 $\mathrm{GeV} / \mathrm{c}$ at various emission angles of neutrons. Points are experimental data [4]. Lines are calculations by the FTFP, FTFB, Bertini and Binary cascade models

The FTF+Preco (FTFP), Bertini, Binary cascade model (BIC) and FTF+Binary cascade model (FTFB) calculations are also given. Preco and Binary Cascade models were added to the FTF model for the simulation of secondary slow particles propagation inside the target nucleus. The Preco model considers absorptions of the secondary slow particles. The Binary Cascade model takes into account all cascading processes and absorptions of the secondary slow particles. Due to this, FTFP is faster than FTFB. As seen in Fig. 2, the FTFB calculations (gray lines) are above the experimental data. The Binary Cascade model calculations (green lines) underestimate neutron production at large angles. The FTFP (red lines), Bertini (blue lines) model calculations are close to each other and describe the experimental data quite well. The same good agreement between the experimental data and FTFP as well as Bertini model calculations was obtained for $\mathrm{p}+\mathrm{Al}$ interactions. When we consider neutron spectra in the interactions of proton with lead nuclei at energy $3 \mathrm{GeV}$, all four models - FTFP, FTFB, Bertini and Binary cascade - show quite good agreement with the experimental data. We can conclude that the FTFP model works well for proton interactions with light and heavy nuclei at energy $3 \mathrm{GeV}$ and higher (see above).

We also tested the FTFP model at lower energies. For this, the experimental data on neutron production by 1.2 and $1.6 \mathrm{GeV}$ protons on $\mathrm{Fe}$ and $\mathrm{Pb}$ nuclei presented in the paper [3] were considered. In Fig. 3, we present the double differential cross sections of neutron production in $\mathrm{p}+\mathrm{Pb}$ interactions as functions of neutron kinetic energy at various angles of emitted neutrons, from 0 to 160 degrees. We compare the experimental data with results of 
the FTFP, FTFB, Bertini and Binary cascade models. At larger angles, FTFP overestimates the neutron spectra at energy larger than $40 \mathrm{MeV}$. The Binary cascade model underestimates production of neutrons in backward hemisphere and overestimates experimental data at small angles 0,10 degrees. The Bertini model calculations are quite close to the experimental data. At small and intermediate angles, the FTFP describes the most part of the experimental data. The FTFB results are worse than the FTFP results. FTFB underestimates neutron yield at small angles less than 30 degrees and overestimates the production of energetic neutrons for angles more than 90 degrees. We obtained similar results for neutron spectra for lead target at the energy $1.2 \mathrm{GeV}$ and for iron target at the energies 1.2 and $1.6 \mathrm{GeV}$. On the whole, we can conclude that the FTFP model describes satisfactorily neutron spectra in proton-nucleus interactions at initial energy more than $1 \mathrm{GeV}$. Let us turn to neutron produc-
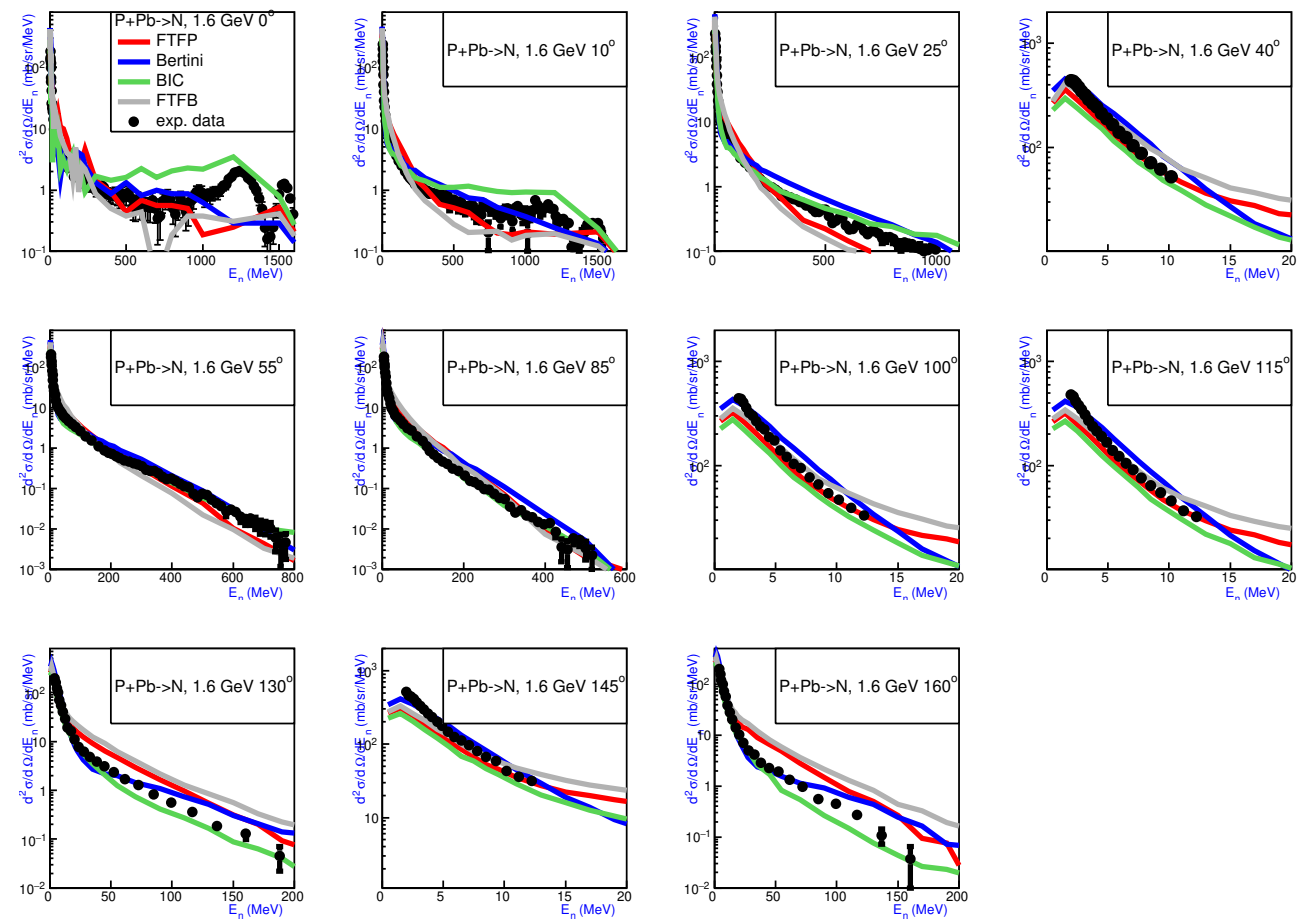

Figure 3. Inclusive cross sections of neutrons produced in $\mathrm{p}+\mathrm{Pb}$ interactions at projectile energy 1.6 $\mathrm{GeV}$ at various emission angles of neutrons. Points are the experimental data [4]. Lines are calculations by the FTFP, FTFB, Bertini, Binary cascade models

tion in antiproton-nucleus interactions. For the simulation of antiproton-proton interactions, the FTF model uses the main assumptions of the Quark-Gluon-String Model [16]. The FTF model assumes production and fragmentation of quark-anti-quark and diquark-anti-diquark strings in the mentioned interactions. The main ingredients of the FTF model are cross sections of string creation processes [15] and application of the LUND string fragmentation algorithm. In antiproton-nucleus interactions, neutrons can be produced at diquark-anti-diquark string fragmentation, and in the processes which we mentioned for proton-nucleus interactions. In Fig. 4, we show kinetic energy distributions of neutrons produced in anti-proton 
interactions with heavy nuclei - Ta and $\mathrm{U}$, at projectile momentum $1.22 \mathrm{GeV} / \mathrm{c}$. We compare the experimental data [5] with calculations by the FTFP and FTFB models.
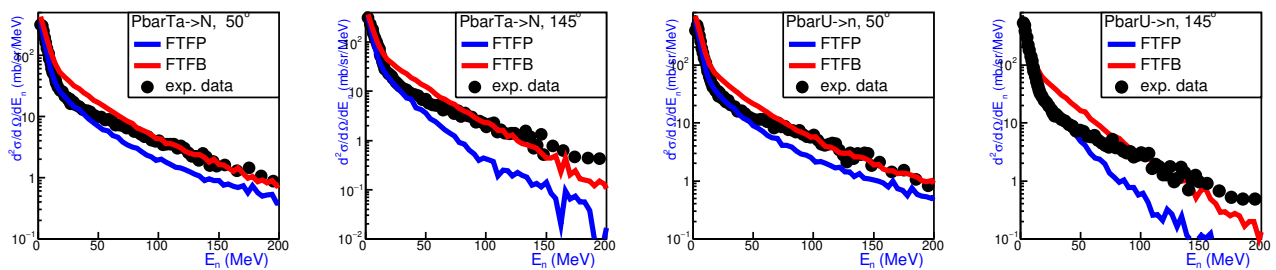

Figure 4. Inclusive cross sections of neutrons produced in antiproton interactions with Ta and $U$ at projectile momentum $1.22 \mathrm{GeV} / \mathrm{c}$. Points are experimental data [5]. Blue and red lines are calculations by the FTFP and FTFB models, correspondingly

The experimental data are given for forward (50 degrees), and backward (145 degrees) angles. The FTFP describes slow neutron production at energy lower than $40 \mathrm{MeV}$ and underestimates fast neutron production. The FTFB results are in reasonable agreement with the experimental data at forward angles. The FTFB model slightly underestimates the production of fast neutrons at backward angles. On the whole, the FTFB model better describes neutron spectra in antiproton-nucleus interactions than FTFP. A correct description of neutron experimental data in the FTF model is very important for the estimation of radiation doses for the future PANDA experiment.

Another set of data, which we used for the FTF model validation, are on neutron production in nucleus-nucleus interaction at energies 1-2 GeV/c/nucleon. The experimental data were obtained at JINR (Dubna) [6]. We compared the FTF model calculations with the experimental data on kinetic energy distributions of neutrons produced in $\mathrm{p}, \mathrm{d}, \mathrm{He}-4, \mathrm{C}+\mathrm{Pb}$ interactions at different angles of emitted neutrons. We obtained that the FTF calculations are in a good agreement with the experimental data for $\mathrm{p}+\mathrm{Pb}$ and $\mathrm{d}+\mathrm{Pb}$ interactions. For $\mathrm{C}+\mathrm{Pb}$ interactions, the FTF model gave larger multiplicity of fast neutrons than the experimental data. It was also demonstrated in [2] that FTFP reproduces spectra of neutrons for carbon interactions with light nuclei $(\mathrm{C}, \mathrm{Al})$. We consider the obtained results as promising ones.
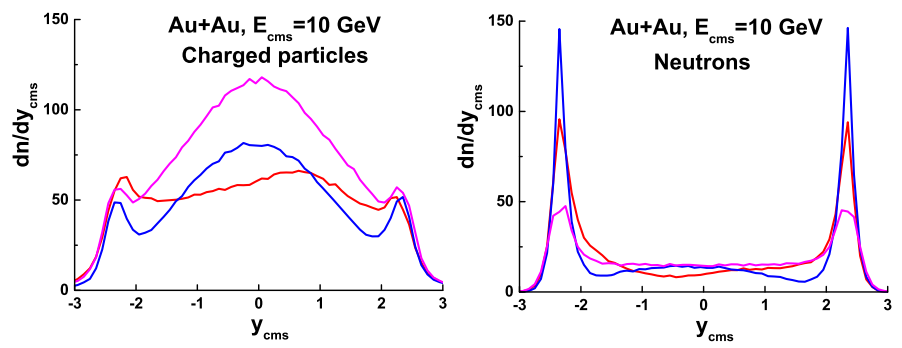

Figure 5. Rapidity distribution of charged particles (left) and neutrons (right) produced in $\mathrm{Au}+\mathrm{Au}$ interactions at NN center of mass energy $10 \mathrm{GeV}$. Blue, red, violet lines are calculations by the FTFP, QGSM (Shield version), UrQMD 1.3, correspondingly

The FTFP model predictions for the NICA/MPD experiment at JINR are presented in Fig. 5. We simulated with the FTFP, UrQMD 1.3, QGSM (SHIELD version) models Au+Au interactions at center of mass energy of NN collisions $10 \mathrm{GeV}$. Charged particle and neutron 
rapidity distributions are plotted in Fig. 5. Large differences between the model calculations are observed for neutron spectra. FTFP predicts multiplicity of neutrons produced in the fragmentation regions higher than UrQMD and QGSM. This difference complicates the design of a forward hadron calorimeter which can be used for centrality determination. The FTF estimation is more realistic than UrQMD and QGSM ones, because we checked the FTF results for neutron spectra in the hadron-nucleus and nucleus-nucleus interactions.

Acknowledgements. A. Galoyan is grateful to heterogeneous computing team of LIT JINR (HybriLIT) for support of calculations and Professor Cheuk-Yin Wong for helpful discussions.

\section{References}

[1] Yu.D. Bayukov et al., ITEP preprint No 172 (1983)

[2] A.S. Galoyan, A. Ribon, and V.V. Uzhinsky, JETP Letters 102, 324 (2015)

[3] S. Leray et al., Phys. Rev. C 65, 044624 (2002)

[4] K. Ishibashi et al., J. Nucl. Sci. Tech. 34, 529 (1997)

[5] T. von Egidy et al., Eur. Phys. J. A 8, 197 (2000)

[6] V.I. Yurevich, R.M. Yakovlev, and V.G. Lyapin, Phys. of Atom. Nucl 69, 1496 (2006). ibig 75, 191 (2012)

[7] S. Bass et al., Prog. Part. Nucl. Phys. 41, 255 (1998). arXiv:nucl-th/9803035 [nucl-th]

[8] M. Bleicher et al., J. Phys. G 25, 1859 (1999). arXiv:hep-ph/9909407 [hep-ph]

[9] https://mcnp.lanl.gov/

[10] http://www.fluka.org/fluka.php

[11] J.Allison et al., Nucl. Instrum. Meth. A 835, 186 (2016)

[12] B. Andersson et al., Nucl. Phys. B 281, 289 (1987)

[13] B. Nilsson-Almquist and E. Stenlund, Comp. Phys. Commun. 43, 387 (1987)

[14] K. Abdel-Waged, V.V. Uzhinsky, Phys. Atom. Nucl. 60, 828 (1997)

[15] A. Galoyan, A. Ribon and V.Uzhinsky, PoS Baldin ISHEPP XXII, 049 (2015)

[16] A. Capella, U. Sukhatme, C.-I. Tan, J. Tran Thanh Van, Phys. Rept. 236, 225 (1994) 